\title{
Optical Absorption in Nano-Structures: Classical and Quantum Models
}

\author{
Anand Kulkarni, Durdu Guney, and Ankit Vora \\ Department of Electrical and Computer Engineering, Michigan Technological University, Houghton, MI 49931, USA
}

Correspondence should be addressed to Durdu Guney; dguney@mtu.edu

Received 11 September 2013; Accepted 7 October 2013

Academic Editors: M. R. Ferreira and M. Mirzaei

Copyright (C) 2013 Anand Kulkarni et al. This is an open access article distributed under the Creative Commons Attribution License, which permits unrestricted use, distribution, and reproduction in any medium, provided the original work is properly cited.

\begin{abstract}
In the last decade, nano-structured materials have gained a significant interest for applications in solar cells and other optical and opto-electronic devices. Due to carrier confinement, the absorption characteristics in these structures are quite different from the absorption in bulk materials and thin films. Optical absorption coefficients of a silicon nano-wire are obtained based on a semiclassical model where the photon-electron interaction is described by the interaction of an electromagnetic wave with the electrons in the valence band of a semiconductor. The absorption characteristics showed enhanced optical absorption but no resonant peaks. In our modified model, we have identified optically active inter band transitions by performing electronic structure calculations on unit cells of nano-dimensions. The absorption spectrum obtained here shows explicit excitonic processes. This absorption is tunable from the visible region to near UV portion of the solar spectrum. In our previous work on thin films (100 nm) of ITO, we have used classical Drude model to describe free electron absorption. Using the imaginary part of the calculated complex dielectric function, we have plotted the absorption coefficient versus wavelength of the photon and compared with the experimental data showing good agreement between theory and experiment.
\end{abstract}

\section{Introduction}

The efficiency of a solar cell is dependent on the optical absorption of the material used to fabricate the solar cell. In bulk crystalline materials and even in thin films, the bulk absorption coefficient is the most important parameter that determines the optical absorption. Classical models such as Drude model or Drude-Lorentz model describe the optical absorption based on the complex dielectric function $[1,2]$. This approach works very well for the absorption of photons by the electrons inside a band, for example, conduction band. However, from band to band excitation of electrons, a detailed understanding of the band structure of the material is essential. To calculate band structure in semiconductors, one has to invoke quantum mechanical models using Schrodinger's wave equation $[3,4]$. In this paper, we illustrate the applications of classical and quantum models with examples from our past research on indium tin oxide (ITO) thin films [5, 6], silicon quantum dots [7], and silicon nanowires [8]. In nanostructures, excitons play a dominant role $[9,10]$.

\section{Classical Models}

2.1. Drude Model. When the energy of incident radiation is less than the band gap energy of a semiconductor (typically $1 \mathrm{eV}$ ), free carrier absorption (electrons in the conduction band and holes in the valence band) takes place. The electric fields of the incident photons accelerate the electrons, which in turn are decelerated by collisions with the phonons, the quantized vibrations of the lattice.

Using Maxwell's equations, it is possible to derive an expression for the complex dielectric function [11]:

$$
\begin{aligned}
\varepsilon^{*}(\omega) & =(\eta-j k)^{2}=\varepsilon_{1}-j \varepsilon_{2} \\
& =\varepsilon_{\infty}-\frac{n q^{2}}{\varepsilon_{0} m_{n}^{*}\left(\omega^{2}+j(\omega / \tau)\right)} .
\end{aligned}
$$

Here $\eta$ and $k$ are the refractive index and extinction coefficient, respectively. $\varepsilon_{\infty}$ is the high-frequency dielectric constant, $n$ is the free electron concentration, $q$ is the electron 
charge, $m_{n}^{*}$ is the effective mass, and $\tau$ is the relaxation time. The imaginary part of $\varepsilon^{*} \equiv \varepsilon_{2}$ is obtained from (1) as

$$
\varepsilon_{2}(\omega)=2 \eta k=\frac{n q^{2}}{\varepsilon_{0} m_{n}^{*} \omega \tau\left(\omega^{2}+1 / \tau^{2}\right)} .
$$

Defining plasma frequency, $\omega_{p}$, as

$$
\omega_{p}^{2}=\frac{n q^{2}}{\varepsilon_{0} m_{n}^{*}}
$$

an expression for the extinction coefficient, $k$ is obtained from (1), (2), and (3):

$$
k^{2}=\frac{1}{2}\left[\left(\varepsilon_{\infty}-\beta\right)^{2}+\frac{1}{\omega^{2} \tau^{2}}(\beta)^{2}\right]^{1 / 2}+\frac{1}{2}\left[\varepsilon_{\infty}-\beta\right],
$$

where $\beta=\omega_{p}^{2} /\left(\omega^{2}+1 / \tau^{2}\right)$; if $\omega_{p} \ll \omega, \beta \ll 1$, at extremely high frequencies, $k^{2} \cong \varepsilon_{\infty}$ as expected and if $\omega \ll \omega_{p}$ and $\omega \tau \ll 1$, then $\beta \simeq \omega_{p}^{2} \tau^{2}$. If $\beta \gg 1$ then,

$$
k^{2} \approx \frac{\omega_{p}^{2} \tau}{2 \omega} .
$$

As an example, let us consider an ITO film having $n=$ $4.6 \times 10^{20} \mathrm{~cm}^{-3}$. Assuming $m_{n}^{*}=0.3 m_{0}, m_{0}$ being free electron mass, $\varepsilon_{\infty}=4.0$ and $\tau=8.53 \times 10^{-15}$ s (i.e., $\left.\mu=50 \mathrm{~cm}^{2} \mathrm{~V}^{-1} \mathrm{~s}^{-1}\right)$, the plasma frequency, $\omega_{p} \approx 2.207 \times$ $10^{15} \mathrm{~s}^{-1}$ or $f_{p}=3.513 \times 10^{14} \mathrm{~s}^{-1}$ is in the infrared region. However, for metals, the plasma frequency can be in the visible or ultraviolet region because of very high free carrier concentration. The absorption coefficient, $\alpha$, is related to the extinction coefficient, $k$, by a simple equation:

$$
\alpha=\frac{4 \pi k}{\lambda}
$$

It is to be noted that $\omega_{p}$ is proportional to $(n)^{0.5}$ and can be controlled from $1.03 \times 10^{14} \mathrm{~s}^{-1}$ to $1.03 \times 10^{16} \mathrm{~s}^{-1}$ by changing $n=1.0 \times 10^{24} \mathrm{~m}^{-3}$ to $n=1.0 \times 10^{28} \mathrm{~m}^{-3}[12]$.

Figure 1 shows a plot of estimated and measured absorption coefficient versus wavelength for an ITO film. As seen in this figure, the calculated plot compares well with the measured data of absorption coefficient for ITO films by Steckl and Mohammed [13]. However, the agreement of the experimental data with the classical Drude theory may deviate because of the following reasons.

(1) Drude theory assumes relaxation time, $\tau$, independent of electron energy.

(2) When photon energies are large compared to the electron energies, quantum models are applicable and these results predict a $\lambda^{3}$ dependence rather than $\lambda^{2}$ dependence as predicted by the Drude theory [2].

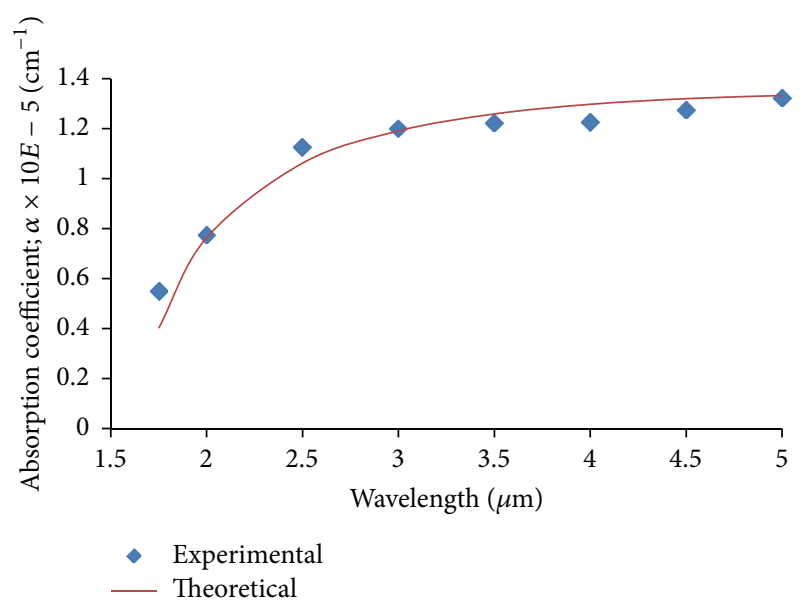

Figure 1: Plot of estimated and experimental [13] absorption coefficient versus wavelength for an ITO film.

\section{Quantum Models}

3.1. Bulk Material and Thin Films. To determine band to band absorption in a semiconductor, one solves time dependent Schrödinger equation:

$$
\left(H_{0}+H^{\prime}\right) \Psi=j \hbar \frac{\partial \Psi}{\partial t}
$$

where $H_{0}$ is the unperturbed Hamiltonian of the system and $H^{\prime}$ corresponds to electron-photon interaction. Representing the photon by a vector potential in the form of a plane electromagnetic wave,

$$
\vec{A}=\frac{1}{2} A \widehat{a} e^{(j(\vec{q} \cdot \vec{r}-\omega t))}+\frac{1}{2} A \widehat{a} e^{(-j(\vec{q} \cdot \vec{r}-\omega t))},
$$

where $\widehat{a}$ is the unit polarization vector in the direction of $\vec{E}$, and $\vec{q}$ is the wave vector of the photon. Using this approach, one solves the transition probability of a transition from state $o$ to state $m$ given by $\left|A_{m}(t)\right|^{2}[4]$ as

$$
\left|A_{m}(t)\right|^{2}=\frac{2 \pi\left|H_{m o}\right|^{2} t}{\hbar} \delta\left(E_{m}-E_{o}-\hbar \omega\right) .
$$

Equation (9) shows clearly that the probability of an electron making a transition from state $o$ with energy $E_{o}$ to a state $m$ with energy $E_{m}$ is zero unless the photon energy $\hbar \omega$ is equal to the difference in energy between the states, thus conserving energy. Also the transition probability is directly proportional to time $t .\left|H_{m o}\right|$ is the time independent matrix element for a given transition from state $o$ to state $m$.

The total probability of the transition $P$ and the transition probability rate $r$ (probability per unit volume per unit time) are given by

$$
\begin{gathered}
P=\frac{2 V}{(2 \pi)^{3}} \int_{\Omega_{k}}\left|A_{k^{\prime}}(t)\right|^{2} f_{o}\left(1-f_{o}\right) d \vec{k}, \\
r=\frac{P}{V t}=\frac{2}{(2 \pi)^{3}} \int_{\Omega_{k}} \frac{\left|A_{k^{\prime}}(t)\right|^{2}}{t} f_{o}\left(1-f_{o}\right) d \vec{k},
\end{gathered}
$$


where $V$ is the crystal volume and $f_{o}$ is the Fermi Dirac distribution function. Expressing $\left|A_{k^{\prime}}(t)\right|^{2}$ as a sum of allowed and forbidden transitions, it is possible to reduce the allowed Hamiltonian to $-(q A / 2 m)\left(\vec{a} \cdot \vec{p}_{k k^{\prime}}\right)$. Thus, $r_{\text {allowed }}$ is given by

$$
r_{\text {allowed }}=\frac{q^{2} A^{2} \omega O_{s}\left(2 m_{r}\right)^{3 / 2}}{8 \pi \hbar^{3} m} f_{o}\left(1-f_{o}\right) \sqrt{\hbar \omega-E_{g}},
$$

where $O_{s}$ is the oscillator strength, a dimensionless quantity defined as

$$
O_{s}=\frac{2\left(\vec{a} \cdot \vec{p}_{k k^{\prime}}\right)}{\hbar m \omega} .
$$

This oscillator strength for a given transition can vary from very low values $\left(10^{-5}\right)$ to 1 depending upon the selection rules. It is significantly higher in direct semiconductors like GaAs. Absorption coefficient, $\alpha_{d}$, is defined as the transition rate per unit quantum flux (quantum flux is defined as the number of incident photons per unit time). $\alpha_{d} \equiv \alpha$ for direct band gap semiconductor,

$$
\alpha_{d}={\frac{q^{2} O_{s}\left(2 m_{r}\right)^{3 / 2}}{4 \pi \varepsilon_{0} c \eta \hbar^{2} m}}^{f_{o}}\left(1-f_{o}\right) \sqrt{\hbar \omega-E_{g}}
$$

where $m_{r}$ is the reduced mass and $\eta$ is the refractive index. Substituting the values for the constants for GaAs, we get

$$
\alpha_{d}(\hbar \omega)=\frac{1.5 \times 10^{5} \sqrt{\hbar \omega-E_{g}}}{\eta(\lambda)}\left(\mathrm{cm}^{-1}\right) .
$$

Figure 2 shows a plot of $\alpha_{d}(\hbar \omega)$ versus $\hbar \omega$ along with an experimental plot for GaAs. A very good agreement is seen between theory and experiment at low photon energies and the discrepancy between experimental data and theory at high photon energies may be due to free carrier absorption as well.

3.2. Nanostructures. In order to improve the efficiency of a solar cell, one obvious choice is to use multiple band gap devices to enable absorption of maximum number of photons of the solar spectrum. These devices also called "tandem" solar cells are discussed in the literature $[14,15]$. Here the complexity of materials used, the compatibility of the adjacent layers, and the manufacturing difficulties lead to very expensive devices for increased efficiency performance.

An alternative way to boost optical absorption is to use nano structure based devices to attain multiple band gaps based on the size of the quantum dots or quantum wells (based on quantum mechanics, the size of the dot or well determines the band gap of the material). Since the nanostructures of silicon result in direct band gap material, the optical absorption is enhanced due to an increase of oscillator strength. We assumed a value of one for the oscillator strength of nanostructured silicon and the reduced mass is taken as half the mass of electron rest mass. The band gap energy is taken as $1.82 \mathrm{eV}$ for a cluster of 18 atoms (a quantum dot of radius $1 \mathrm{~nm}$ ) [16]. Plugging the numbers in

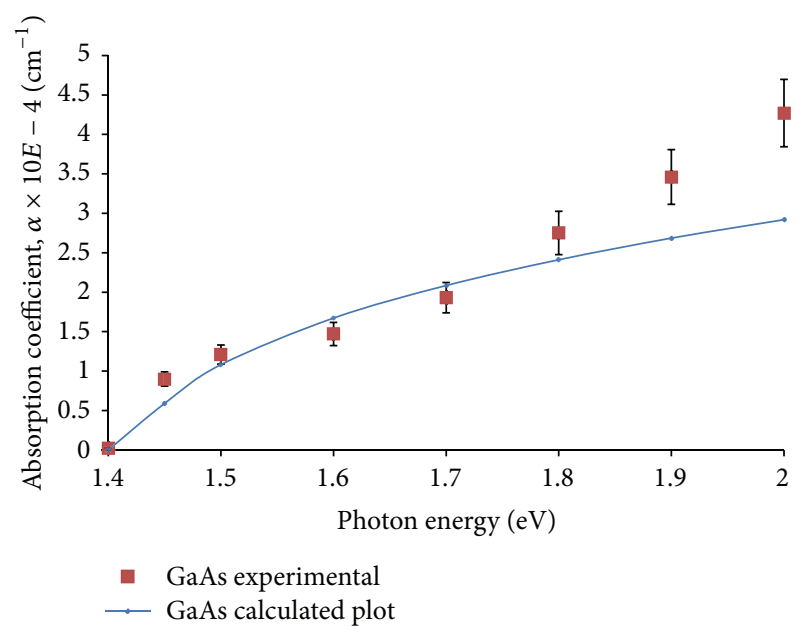

Figure 2: Plot of $\alpha_{d}(\hbar \omega)$ versus $\hbar \omega$ along with an experimental [2] plot for GaAs.

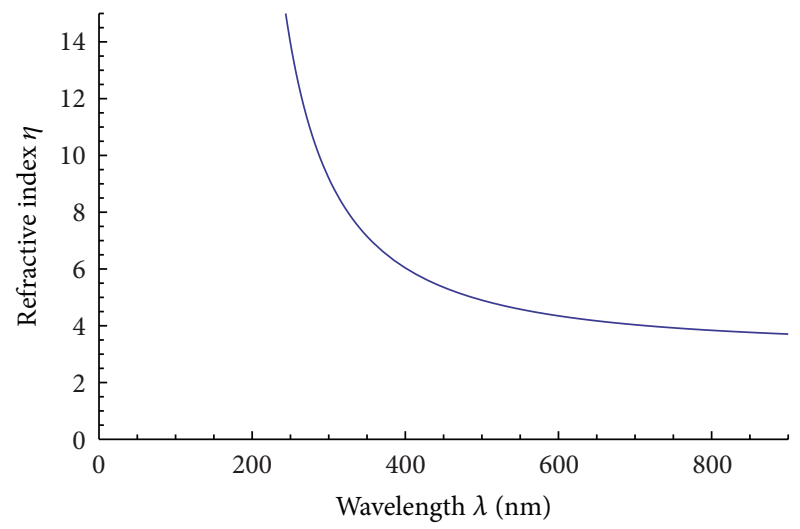

Figure 3: Plot of $\eta(\lambda)$ versus wavelength for silicon.

(13), we get an expression for the absorption coefficient for nano structured silicon as

$$
\alpha_{d} \cong \frac{5.4 \times 10^{5}}{\eta(\lambda)}\left[\frac{1.24}{\lambda}-1.82\right]^{1 / 2}\left(\mathrm{~cm}^{-1}\right),
$$

where $\lambda$ is the incident photon wavelength in $\mu \mathrm{m} . \eta(\lambda)$ is the refractive index given by Herzberger's formula.

$$
\eta(\lambda)=3.2346+\frac{0.3698}{\lambda^{2}-0.028} .
$$

Figure 3 shows a plot of $\eta(\lambda)$ versus $\lambda$ based on (16) and Figure 4 shows a plot of $\alpha_{d}$ versus $\lambda$ for nanostructures of bandgap varying from $1.69 \mathrm{eV}$ to $3.65 \mathrm{eV}$ (quantum dot size varies from $2 \mathrm{~nm}$ to $7 \mathrm{~nm}[17,18]$ ). As seen in Figure 4, the absorption coefficient, $\alpha$ quickly increases to $1 \times 10^{5} \mathrm{~cm}^{-1}$ for a quantum dot having $E_{g}=1.69 \mathrm{eV}$. Comparing $\alpha$ values in bulk silicon (less than $10^{4} \mathrm{~cm}^{-1}$ ), we observe an order of magnitude enhancement in $\alpha$. Smith et al. have performed time resolved PL measurements on silicon nano-particles ( $1 \mathrm{~nm}$ ) and have reported direct band to band transition with emission in the wavelength range of $400-480 \mathrm{~nm}$ supporting our estimated results [19]. 


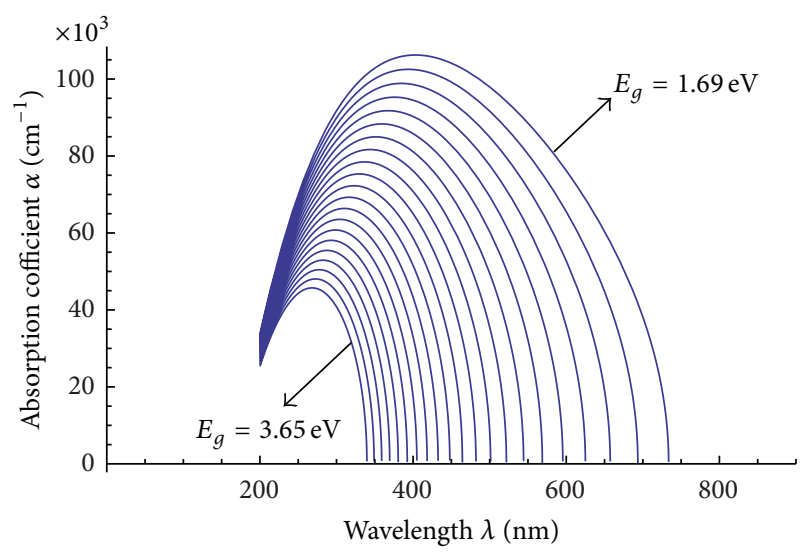

FIGURE 4: Plot of $\alpha_{d}$ versus $\lambda$ for nanostructures of bandgap varying from $1.69 \mathrm{eV}$ to $3.65 \mathrm{eV}$.

TABLE 1: Radiative lifetime of lowest optically active excitons.

\begin{tabular}{lccccccc}
\hline System & $\varepsilon_{1}(0)$ & $\eta$ & $E_{b}(\mathrm{ev})$ & $\lambda_{f}(\mathrm{~nm})$ & $m_{e}^{*}$ & $O_{s}$ & $\tau(\mu \mathrm{s})$ \\
\hline Sil1-a & 1.5 & 1.2 & 0.1655 & 2.55 & 1.92 & 0.016 & 0.152 \\
Sil1-b & 1.5 & 1.2 & 0.2068 & 3.457 & 0.58 & 0.003 & 0.198 \\
Sil1-c & 1.5 & 1.2 & 0.188 & 3.1 & 0.91 & 0.233 & 0.004 \\
Si22 & 3 & 1.7 & 0.1314 & 4.216 & 0.71 & 0.051 & 0.022 \\
Si33 & 5 & 2.4 & 0.1095 & 5.08 & 0.58 & 0.0014 & 0.955 \\
\hline
\end{tabular}

These authors also note that polysilane displays strong absorption from 280 to $350 \mathrm{~nm}$ to further substantiate our theoretical plots. The decrease of absorption coefficients for low values of wavelength is related to significant increase in refractive index with decreasing wave lengths in bulk silicon which may not be true in silicon nanostructures.

3.3. Revised Band Structures. In order to improve our quantum mechanical model for nanostructures, we performed electronic structure calculations on unit cells of nano dimensions and determined optically active interband transitions. The computations were carried out using a density functional DFT-LDA approach using a parallel binary of the PWScf distribution. Figure 5 shows silicon nanowire unit cell geometries seen along [100] direction. Three nanowire unit cells with a diameter $0.434 \mathrm{~nm}, 0.816 \mathrm{~nm}$, and $1.075 \mathrm{~nm}$ have been designated as Sill, Si22, and Si33 systems. The details of the calculations and computed oscillator strengths for direct $\vec{k}$ transitions can be found in another paper [8]. In summary, silicon nanowires exhibited direct band gaps as observed by several other research groups [20-22]. The energy band structures for these systems are shown in Figure 6. Exhibiting dispersion curves with very small slopes, these curves yield exceptionally high effective masses for electrons and holes (see Table 1). The absorption spectrum of these nanostructures shows explicit excitonic processes and these processes are described in detail below.

\section{Excitonic Processes}

Because of the confinement of electrons and holes in nanostructures, the photon energy absorbed will result into excitons with significant binding energies (in $\mathrm{eV}$ ) and appreciable life times (in $\mu \mathrm{s}$ ). We have evaluated the dependence of the binding energy $\left(E_{b}\right)$ of 1-D exciton on the length of a nanowire. Following the methods outlined in $[23,24]$, solving a two particle Schrödinger wave equation for an electron-hole system, and using approximate Hamiltonian, $H$, and the wave function, $\Psi$, and a variational method of minimizing the total energy over different values of $\lambda$ (a fitting constant of $\Psi$ ), the ground state of exciton binding energy is calculated as

$$
E_{b}=\min _{\lambda} \frac{\langle\Psi|H| \Psi\rangle}{\langle\Psi \mid \Psi\rangle} .
$$

The minimum value of $\lambda$ corresponds to the effective Bohr radius of the exciton. The binding energies have been plotted on a function of nanowire length in Figure 7. The binding energies monotonically increase as the nanowire is made shorter. This dependence is expected as the strong confinement causes an increase in the effective mass of the charge carriers. The bulk excitonic binding energy is dependent on the reduced mass $\mu$ of the exciton given by

$$
E_{\text {bulk }}=\frac{\mu q^{4}}{3 \pi^{2} \hbar^{2} \varepsilon_{0}^{2} \varepsilon_{r}^{2}},
$$

where $\mu=m_{e} m_{h} /\left(m_{e}+m_{h}\right)$. The typical Bohr radii for the $1 \mathrm{D}$ free exciton, $\lambda_{f}$, was found to range between 2.5 and $5 \mathrm{~nm}$. The radiative life time, $\tau_{r}$, is related to the oscillator strength $O_{s}$ and the exciton binding energy $\left(E_{b}\right)$ by the relation $[25$, 26]:

$$
\frac{1}{\tau_{r}}=\frac{n q^{2} E_{v} O_{s}}{2 \pi \varepsilon_{0} m_{e} \hbar^{2} c^{3}}
$$

where $E_{v}=E_{g}-E_{b}$. These nanowires exhibit unusually large exciton life times in the order of a microsecond. The results for a specimen of $10 \mathrm{~nm}$ are summarized in Table 1. As seen in this table, $E_{b}$ gradually increases from Si33 to Sill due to a decrease of screening potential with a decrease of nearest neighbors. As $E_{b}$ increases, $E_{g}-E_{b}$ decreases, decreasing $1 / \tau_{r}$, thus increasing the radiative life time as expected. However, a large oscillator strength (determined by selective rules) may result into a quicker decay as seen is Sillc compared to Silla and Sillb.

The $\alpha(\omega)$, absorption coefficients for Sil1, Si22, and Si33, are shown in Figure 8. The lowest excitonic peaks for each of the nanowires occur at $5.25 \mathrm{eV}(232 \mathrm{~nm}), 3.7 \mathrm{eV}(335 \mathrm{~nm})$, and $2.3 \mathrm{eV}(539 \mathrm{~nm})$ in the increasing order of size. The peak absorption coefficients range from $2 \times 10^{7} \mathrm{~m}^{-1}$ for Sill to $15 \times 10^{7} \mathrm{~m}^{-1}$ for Si33. These values are approximately an order of magnitude higher compared to $\alpha_{d}$ versus $\lambda$ shown in Figure 4, where the excitonic effects are not included. Note that absorption is tunable from visible region to the near UV portion of the solar spectrum. The prominent peaks seen in Si22 wire are less prominent in Si33 wire since the density of states in conduction band of Si33 increases (compared to 


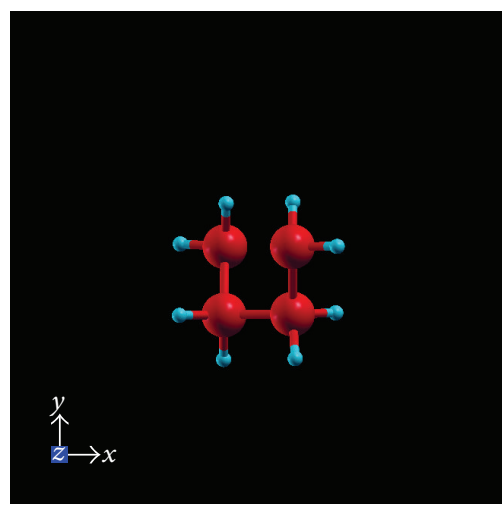

(a)

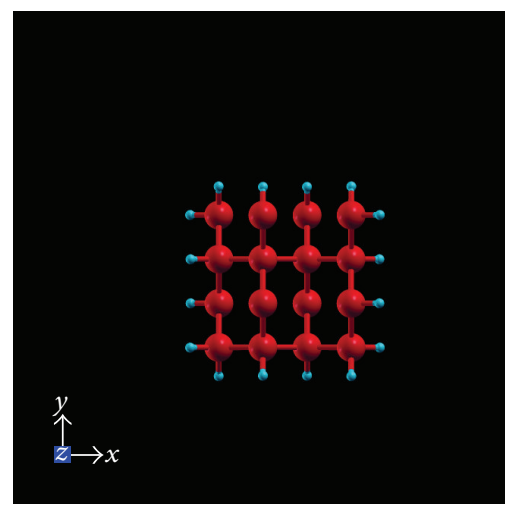

(b)

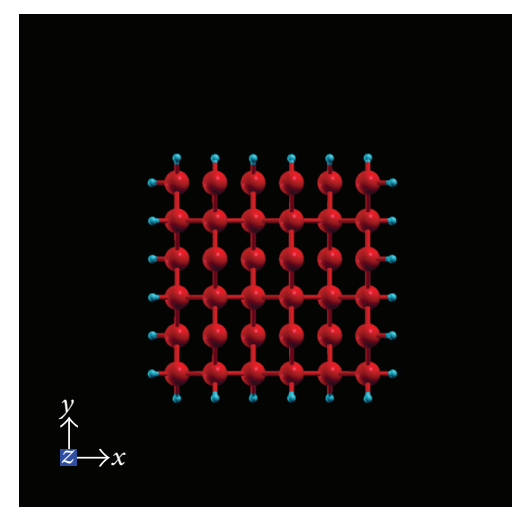

(c)

FIGURE 5: Silicon nanowire unit cell geometries seen along [100] direction. Three nanowire unit cells with a diameter $0.434 \mathrm{~nm}, 0.816 \mathrm{~nm}$, and $1.075 \mathrm{~nm}$ have been designated as (a) Sil1, (b) Si22, and (c) Si33.

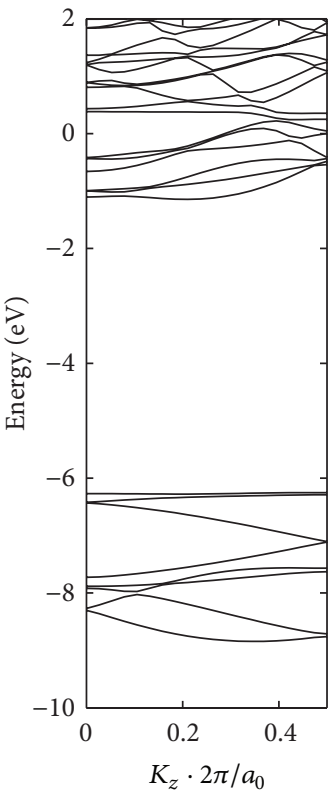

(a)

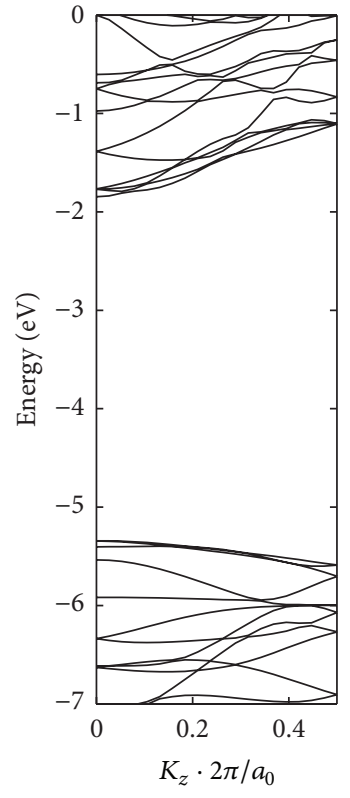

(b)

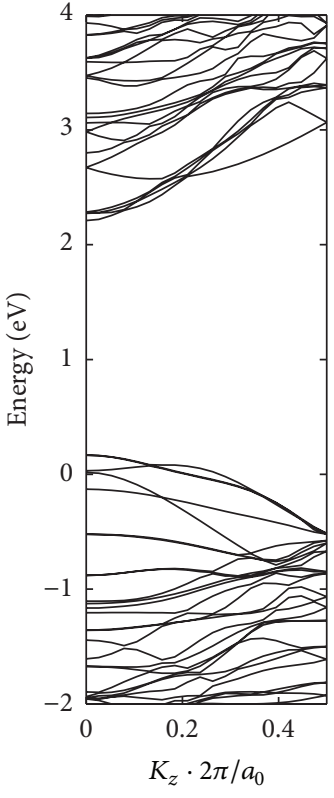

(c)

FIGURE 6: 1-D band-structure of silicon nanowire system along the $z$-direction with $0<k z<\pi / a_{0}$ with $a_{0}=5.43 \AA$ being the lattice constant. Band diagrams are shown for (from left to right) Sil1, Si22, and Si33 systems.

Si22), thus decreasing the resonance effects. Also the rippled nature of the absorption spectrum is less in Si33 compared to Si22. Thus, the absorption spectra of nanostructures are dominated by the excitonic processes.

\section{Conclusions}

Drude model gives a fairly good explanation of the optical absorption by free carriers. Though one could describe the excitations of free electrons in the conduction band using quantum mechanics, the advantages are valid when photon energies are significantly higher than electron energies. For band to band transition, the quantum mechanical models depicting the details of the band structures are essential to fully explain the observed absorption spectrum in bulk and thin film materials. This approach is not adequate to explain the optical absorption spectra in nanostructures which show resonant peaks and enhanced absorption. Here electronic structure calculations on unit cells of nano dimensions lead to excitonic processes that have a significant impact on absorption. The $E$ versus $\vec{k}$ curves show weak dispersion in nanostructures compared to those of bulk materials resulting in heavier effective masses for both electrons and holes. The binding energies of excitons in nanostructures are a few 


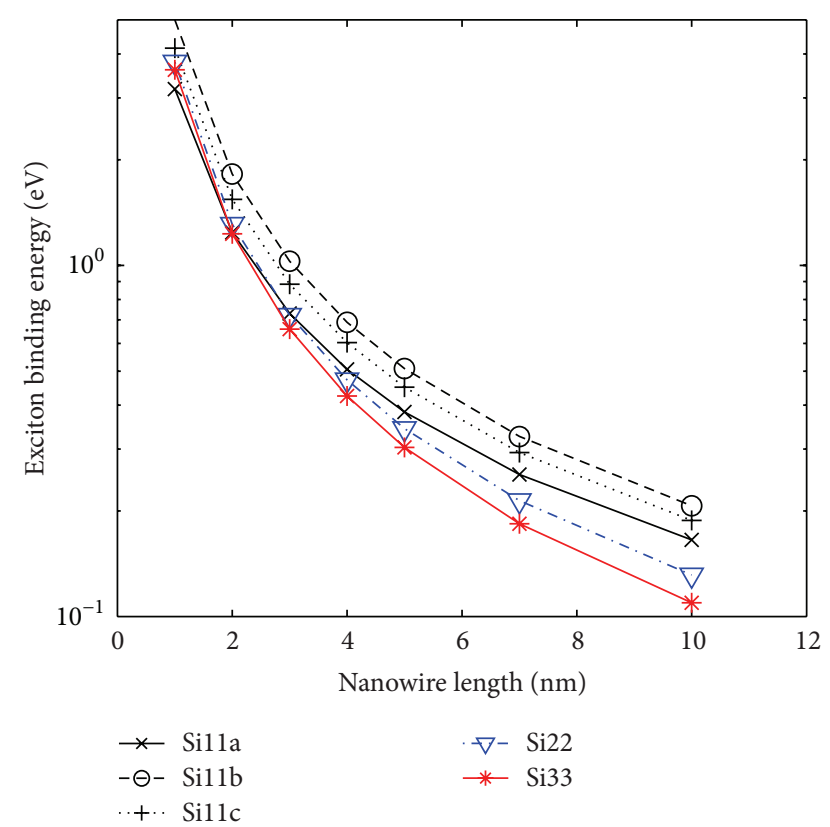

FIgURE 7: 1-D exciton binding energy in silicon nanowires as a function of nanowire length.

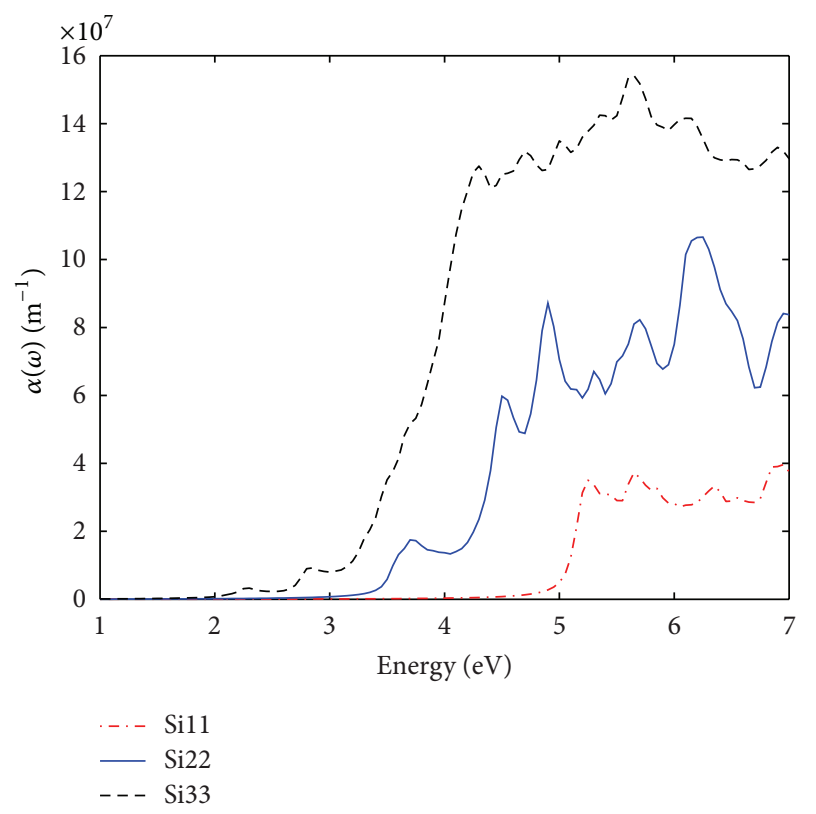

FIGURE 8: Optical absorption coefficient for the nanowire systems.

tenths of $\mathrm{eV}$ compared to a few hundredths of $\mathrm{eV}$ in bulk materials $(0.015 \mathrm{eV}$ in $\mathrm{Si})$. The radiative life times are in microseconds in nanostructures as compared to nanoseconds as observed in direct bandgap semiconductors, for example, GaAs. In nanostructures, only certain transitions from valence band to conduction band dominate indicating large oscillator strengths, a measure on the strength of absorption for a given energy of photon. Hence, optical absorption in nanostructures is a lot more complex phenomena than that in bulk materials or thin films.

\section{Acknowledgment}

This work was supported by the National Science Foundation under grant CBET-1235750. We also would like to thank Jaspreet Singh and Vidur Parkash for their help with the figures.

\section{References}

[1] P. Y. Yu and M. Cardona, Fundamentals of Semiconductors, Springer, New York, NY, USA, 3rd edition, 2001.

[2] E. D. Palik, Handbook of Optical Constants of Solids, Academic Press, New York, NY, USA, 1985.

[3] R. A. Smith, Semiconductors, Cambridge University Press, New York, NY, USA, 2nd edition, 1978.

[4] C. M. Wolfe, N. Holonyak, and G. E. Stillman, Physical Properties of Semiconductors, Pentice Hall, New Jersey, NJ, USA, 1989.

[5] S. A. Knickerbocker and A. K. Kulkarni, "Estimation and verification of the optical properties of indium tin oxide based on the energy band diagram," Journal of Vacuum Science and Technology A, vol. 14, no. 3, pp. 757-761, 1996.

[6] A. K. Kulkarni and S. A. Knickerbocker, "Estimation and verification of the electrical properties of indium tin oxide based on the energy band diagram," Journal of Vacuum Science and Technology A, vol. 14, no. 3, pp. 1709-1714, 1996.

[7] J. S. Nayyar and A. Kulkarni, "One aspect of solar cell efficiency: multiple wavelengths photon absorption in nano-silicon structures," in Proceedings of the SPIE Nanoscale Photonic and Cell Technologies for Photovoltaics II, vol. 7411, August 2009.

[8] V. Parkash and A. K. Kulkarni, "Optical absorption characteristics of silicon nanowires for photovoltaic applications," IEEE Transactions on Nanotechnology, vol. 10, no. 6, pp. 1293-1297, 2011.

[9] T. Takagahara and K. Takeda, "Theory of the quantum confinement effect on excitons in quantum dots of indirect-gap materials," Physical Review B, vol. 46, no. 23, pp. 15,578-15,581, 1992.

[10] M. Bruno, M. Palummo, A. Marini, R. Del Sole, and S. Ossicini, "From Si nanowires to porous silicon: the role of excitonic effects," Physical Review Letters, vol. 98, no. 3, Article ID 036807, 2007.

[11] P. Y. Yu and M. Cardona, Fundamentals of Semiconductors, Springer, New York, NY, USA, 3rd edition, 2001.

[12] S. A. Knickerbocker, Estimation and verification of electrical and optical properties of indium-tin-oxide based on energy band diagrams [Ph.D. thesis], Michigan Technological University, Houghton, Mich, USA, 1995.

[13] A. J. Steckl and G. Mohammed, "The effect of ambient atmosphere in the annealing of indium tin oxide films," Journal of Applied Physics, vol. 51, no. 7, pp. 3890-3895, 1980.

[14] M. A. Green, Third Generation Photovoltaics: Advanced Solar Energy Conversion, Springer, Berlin, Germany, 2006.

[15] J. Nelson, The Physics of Solar Cells, Imperial College Press, London, UK, 2003.

[16] D. C. Johnson, I. M. Ballard, K. W. J. Barnham et al., "Observation of photon recycling in strain-balanced quantum well solar cells," Applied Physics Letters, vol. 90, no. 21, Article ID 213505, 2007. 
[17] L. Cuadra, A. Martí, and A. Luque, “Type II broken band heterostructure quantum dot to obtain a material for the intermediate band solar cell," Physica E, vol. 14, no. 1-2, pp. 162$165,2002$.

[18] A. Luque and A. Martí, "Ultra-high efficiency solar cells: the path for mass penetration of solar electricity," Electronics Letters, vol. 44, no. 16, p. 943, 2008.

[19] A. Smith, Z. H. Yamani, N. Roberts et al., "Observation of strong direct-like oscillator strength in the photoluminescence of $\mathrm{Si}$ nanoparticles," Physical Review B, vol. 72, no. 20, Article ID 205307, 2005.

[20] E. C. Garnett and P. Yang, "Silicon nanowire radial p-n junction solar cells," Journal of the American Chemical Society, vol. 130, no. 29, pp. 9224-9225, 2008.

[21] V. Sivakov, G. Andrä, A. Gawlik et al., "Silicon nanowirebased solar cells on glass: synthesis, optical properties, and cell parameters," Nano Letters, vol. 9, no. 4, pp. 1549-1554, 2009.

[22] J. P. Perdew and A. Zunger, "Self-interaction correction to density-functional approximations for many-electron systems," Physical Review B, vol. 23, no. 10, pp. 5048-5079, 1981.

[23] Y. Cui, X. Duan, J. Hu, and C. M. Lieber, "Doping and electrical transport in silicon nanowires," Journal of Physical Chemistry B, vol. 104, no. 22, pp. 5215-5216, 2000.

[24] A. Poruba, J. Springer, L. Mullerova et al., "Temperature dependence of the optical absorption coefficient of microcrystalline silicon," Journal of Non-Crystalline Solids, vol. 338-340, no. 1, pp. 222-227, 2004.

[25] P. M. Derlett, T. C. Choy, and A. M. Stoneham, "An investigation of the porous silicon optical absorption power law near the band edge," Journal of Physics, vol. 7, no. 12, pp. 2507-2523, 1995.

[26] G. Bastard, E. E. Mendez, L. L. Chang, and L. Esaki, "Exciton binding energy in quantum wells," Physical Review B, vol. 26, no. 4, pp. 1974-1979, 1982. 

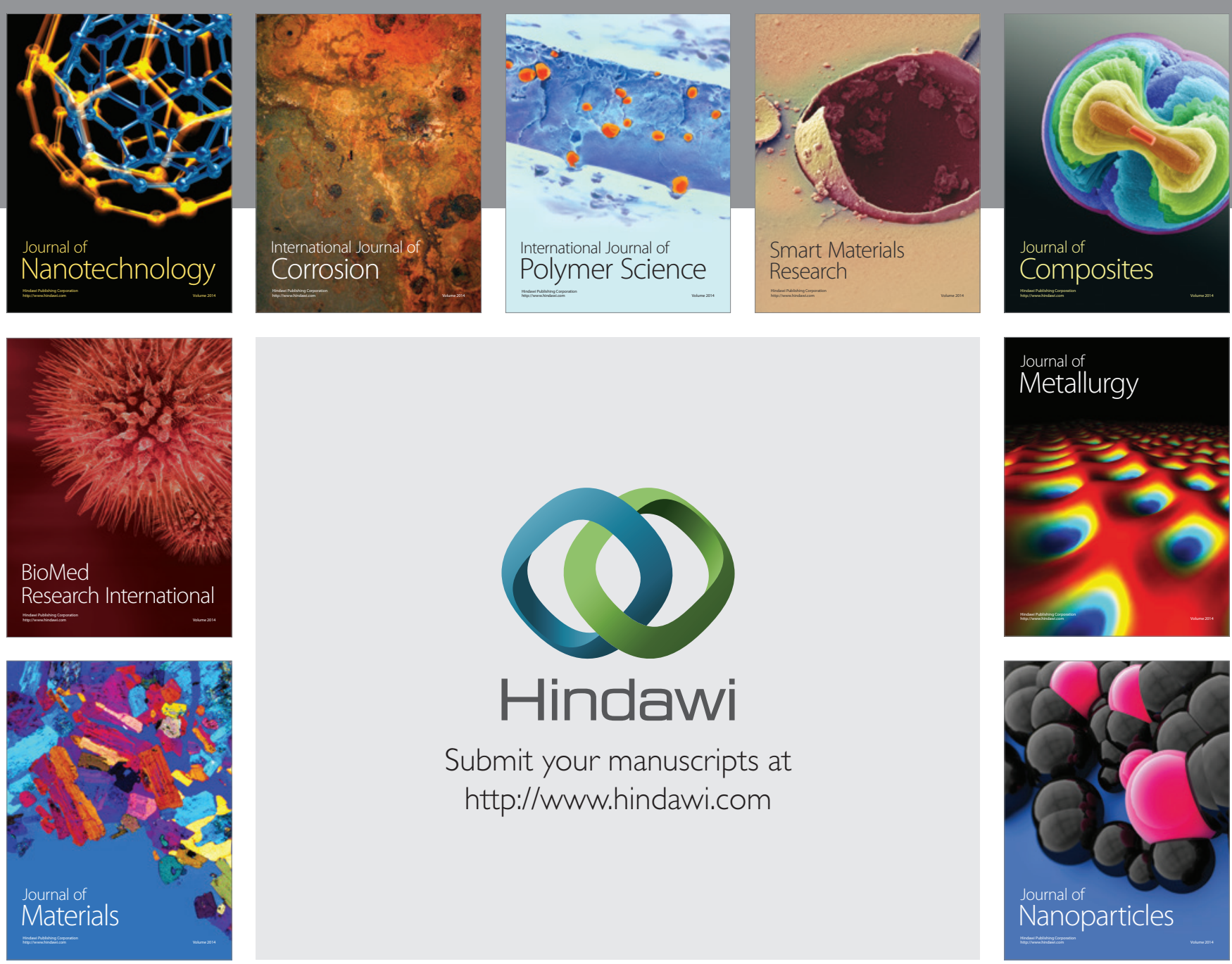

Submit your manuscripts at http://www.hindawi.com
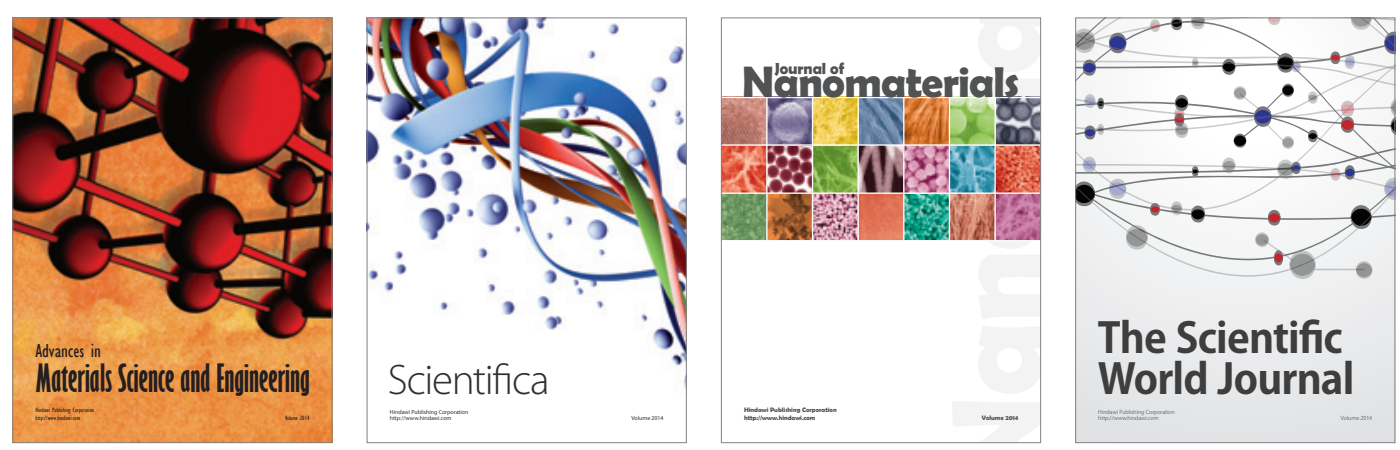

\section{The Scientific World Journal}
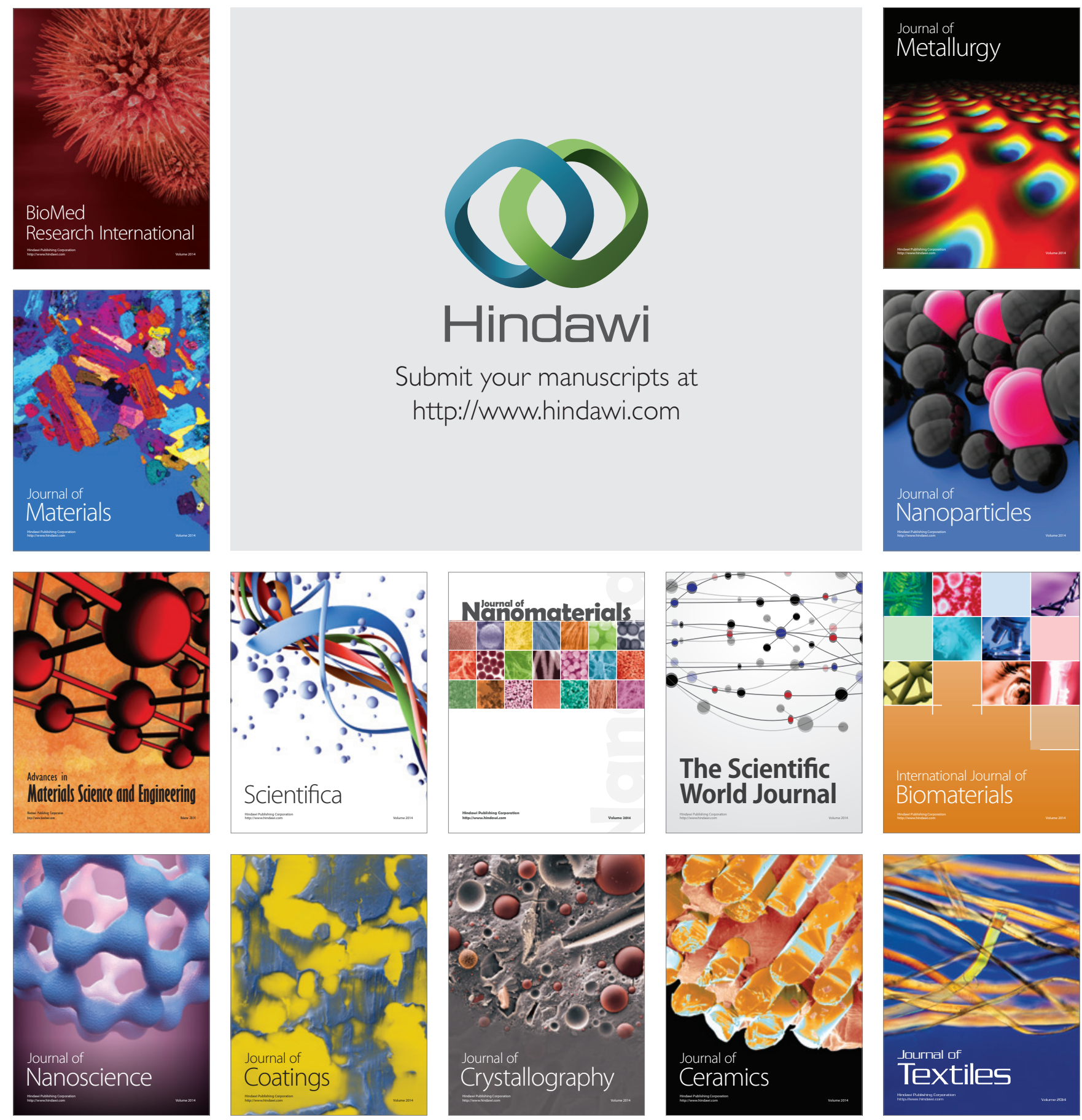